\title{
A NEW PROBABILISTIC ENCRYPTION SCHEME
}

He Jingmin and Lu Kaicheng

Dept. of Computer Science, Isinghua University

Beijing, People's Republic of China

Abstract: In this paper we present a new probabilistic public key cryptosystem. The system is polynomially secure. Furthermore, it is highly efficient in that it's message expansion is $1+(k-1) / 1$, where $k$ is the security parameter and 1 the length of the encrypted message. Finally, the system can be used to sign signatures.

\section{Introduction}

The most important problem in modern cryptography is how to encrypt messages in a secure and efficient way. Here two things are of equal importance: security and efficiency. Up to now three different notions of security have been proposed: Goldwasser and Micali's polynomial security, semantic security [1], and Y-security introduced by Yao [2]. Micali et al([3]) have pointed out that these three notions are essentially equivalent. In this paper we'll adopt the notion of polynomial security. As to the efficiency, it usually means the encrypting and decrypting time and the message expansion.

The earliest public key cryptosystem is RSA [4]. RSA is highly efficient be cause it's message expansion is about one (the possiblely least value). However, it's security remains to be proven. Actually RSA is a deterministic cryptosystem and can't be secure according to [1]. In another direction, Goldwasser and Micali [1] presented the first probabilistic encryption scheme whose polynomial security is rigorously proven. But their scheme is not efficient at all. They encrypt every bit of the message indeperdently, so the message expansion is $k$ (the security parameter) and this makes the scheme totally unvalued in practice.

In this paper we concern both security and efficiency. We present a new "randan iterative encryption scheme" which can achieve both polynomial security and high efficiency. The idea is simple: we randomly and iteratively encrypt the plaintext bit by bit. In this way we can get a secure public key cryptosystem with a low message expansion of $1+(k-1) / 1$, where 1 is the length of the plaintext and $k$ the security par- 
ameter. The one more lucky thing is that the new scheme can be used to sign digital signatures, which seems impossible in the schemes of [1], [5] and [6].

Remark: Blum and Goldwasser have presented another secure probabilistic encryption method with a message expansion of $1+k / 1$. Their method is similar to that of $B 1-$ um et al.([5]), in which it exlusive-or the plaintext with a sequence of the same length generated by a pseudo-random number generator. For the details see [5] and [6].

\section{Background}

Let $N$ denote the set of positive integers and $n \in N$. Let $z_{n}^{*}=\{x \mid 1 \leqslant x<n$ and $(x, n)=1\}, \quad z_{n}^{1}=\{x \mid 1 \leqslant x<n$ and $(x / n)=1\}$, where $(x / n)$ is the Jacobi symbol of $x$ mod $n$. The symbol $|n|$ denotes the binary length of $n$.

Let $Q_{n}$ be a predicate defined on $z_{n}^{1}$ such that $Q_{n}(x)=1$ iff $x$ is a quadratic residue $\bmod n$. Let $H_{k}$ denote the set of "hard composite integers", i.e., $H_{k}=\{n \mid n=p q$, where $p$ and $q$ are distinct primes such that $|p|=|q|=k$. $\}$.

The security of our scheme is based on the quadratic residuosity assumption (QRA). From QRA Goldwasser and Micali have proven the following.

Lemma $1([1])$. Under $Q R A$, the predicate $Q_{n}$ defined on $z_{n}^{1}$ is unappoximable by any circuit of polynomial size even if some quadratic nonresidue mod $n$ are known. (Recall that a circuit $C$-approximates a predicate $Q: B \rightarrow\{0,1\}$ if $C(x)=Q(x)$ for at least a fraction $1 / 2+E$ of the $x \notin B$.

Let $J_{n}=\{x \mid 1<x<n / 2$ and $(x / n)=1\}$. Let $Q R_{n}$ denote the set of quadratic residues mod n. It is easy to prove the following

Lerma 2. Let $n=p q$ where $p$ and $q$ are distinct primes such that $p=q=3$ mod 4 . Then each $z \mathbb{Q R}_{n}$ has exactly one square root that is in $J_{n}$ and we denote this root by $\operatorname{sqr}(z)$.

We point out that Lemma 1 will still hold when $Q_{n}$ defined on $z_{n}^{1}$ is restricted to $\mathrm{J}_{\mathrm{n}}$, and we still call the result Lemma 1.

\section{The New Encryption Scheme}

Let $n=p q$ as in Lema 2 . Let $y$ be a quadratic nonresidue mod $n$. Now we introduce a function $E_{n}$ as follaws:

$$
\begin{aligned}
& E_{n}: J_{n} x\{0,1\} \rightarrow J_{n} x\{0,1\} \\
& E_{n}(x, 0)=\left(x^{2} \bmod n, 0\right) \\
&=\left(-x^{2} \bmod n, 1\right) \\
& E_{n}(x, 1)=\left(x^{2} y \bmod n, 0\right) \\
&=\left(-x^{2} y \bmod n, 1\right)
\end{aligned}
$$

if $x^{2} \bmod n<n / 2$, ocherwise.

if $x^{2} y \bmod n<n / 2$, otherwise.

From Lemma 2 we know that $E_{n}$ is invertible. The inverse of $E_{n}$ is denoted by $D_{n}$ and can be specified as follows: 


$$
\begin{array}{rlrl}
D_{n}: J_{n} \times\{0,1\} \rightarrow J_{n} \times\{0,1\} & & \\
D_{n}(z, j) & =(\operatorname{sqr}(z), 0) & & \text { if } j=0 \text { and } z \in Q R . \\
& =\left(\operatorname{sqr}\left(z y^{-1}\right), 1\right) & \text { if } j=1 \text { and } z \notin Q R . \\
& =\left(\operatorname{sqr}\left(-z y^{-1}\right), 1\right) & \text { if } j=1 \text { and } z \in Q R . \\
& =(\operatorname{sqr}(-z), 0) & & \text { if } j=1 \text { and } z \& Q R .
\end{array}
$$

For convenience we denote the first and second components of $E_{n}(x, i)$ by $E_{n}^{1}(x, i)$ and $E_{n}^{2}(x, i)$ respectively.

For any positive integer $1, E_{n}$ can be generalized as follows:

$$
\begin{aligned}
E_{n}: & J_{n} \times\{0,1\} \stackrel{1}{\longrightarrow} J_{n} \times\{0,1\} \\
& E_{n}\left(x, m_{1} \ldots m_{1}\right)=\left(x_{1}, b_{1} \ldots b_{1}\right)
\end{aligned}
$$

where

$$
\begin{aligned}
& x_{0}=x, \\
& x_{i}=E_{n}^{1}\left(x_{i-1}, m_{i}\right), \\
& b_{i}=E_{n}\left(x_{i-1}, m_{i}\right), \\
& i=1,2, \ldots, 1 .
\end{aligned}
$$

The generalized $E_{n}$ is also invertible and it's inverse is still denoted by $D_{n}$.

Now let $k$ (an even number) be the security parameter. The new probabilistic puolic key cryptosystem works as follows:

(1) it randomly selects two distince primes $p$ and $q$ such that $p=q=3$ mod 4 and $|\mathrm{p}|=|\mathrm{q}|=\mathrm{k} / 2$,

(2) sets $n=p q$,

(3) picks $y$, a quadratic nonresidue $\bmod n$, and finally,

(4) outputs $\{n, y\}$ and $\{p, q\}$.

Some user, say A, publicizes the pair $\{n, y\}$ and keeps secret the pair $\{p, q\}$.

Encryption: Suppose some user $B$ want to send a binary message $m=m_{1} \ldots m_{1}$ to user A. Then he encrypts $m$ as follows:

(1) Randomly selects an $x \in J_{n}$ and sets $z=x$.

(2) Performs step (3) for $i=1,2, \ldots, 1$.

(3) $\left(z, b_{i}\right):=E_{n}\left(z, m_{i}\right)$.

(4) Sends $A$ the ciphertext $E_{n}(x, m)=\left(z, b_{1} \ldots b_{1}\right)$.

Encrypting an 1 -bit long message $m$ takes $O\left(1 k^{2}\right)$ time, and $m$ is transformed into an (1+k-1)-bit long ciphertext. So the message expansion is $1+(k-1) / 1$ which is much less than $k$ (the message expansion of Goldwasser and Micali's scheme).

Decryption: Upon receiving the ciphertext $\left(z, b_{1} \cdots b_{1}\right)$, user A decrypts it as follows :

(1) Performs step (2) for $i=1,1-1, \ldots, 1$.

(2) $\left(z, m_{i}\right):=D_{n}\left(z, b_{i}\right)$.

(3) Gets the message $m=m_{1} \ldots m_{1}$.

Recovering $m(|m|=1)$ from it's ciphertext takes $O\left(1 k^{3}\right)$ time.

Using the proof techniques in [3] and [6], we can prove the following 
Theorem. The cryptosystem introduced above is polynomially secure.

Proof. The proof is tedious long and omitted here.

\section{Applications}

To sign a message $m$, we randomly select $a n x \in J$ and forms

$$
S(m)=\left(m, D_{n}(x, m)\right)
$$

$\mathrm{s}(\mathrm{m})$ will be the signature of $\mathrm{m}$. Of course this simple signature is not strong. By computing $E_{n}(z, b)=(x, m)$, the forger can easily forge the signature of an (unpredictable) message $m$. This is the so-called "chosen signature attack" and can be prevented in several ways. One way is as follows: randonly select $x, y \in J, x \neq y$, and $l e t s(m)=(m$, $\left.D_{n}(x, m), D_{n}(y, m)\right)$. This time forging the signature of even an unpredictable message m requires finding $w, z \in J_{n}, b, b^{\prime} \in\{0,1\}^{*}$, such that $E_{n}(w, b)=E_{n}\left(z, b^{\prime}\right)$, and this seems impossible.

Note that in the above mentioned signature scheme, the signature of $\mathrm{m}_{1} \ldots \mathrm{m}_{i}$ or $m_{i} \ldots m_{1}$ for any $i(1 \leqslant i \leqslant 1)$ can be easily obtained when the signature of $m_{1} \ldots m_{1}$ is known. But we may avoid this danger by letting, for example,

$$
S\left(m_{1} \ldots m_{1}\right)=\left(m_{1} \ldots m_{1}, D_{n}\left(x, m_{1} \ldots m_{1}\right), D_{n}\left(y, m_{1 / 2} \cdots m_{1} m_{1} \cdots m_{\ell / 2+1}\right)\right. \text {. }
$$

Clearly various signature schemes can be devised based on our new public key cryptosystem. We leave the open problem of implementing a concrete signature scheme, together with a rigorious security proof.

\section{References}

[1] S.Goldwasser and S.Micali, Probabilistic encryption, journal of Computer and System Sciences $28(1984), 270-299$.

[2] A.Yao, Theory and application of trapdoor functions, Proceedings of the $23 \mathrm{rd}$ Annual Symposium on Foundations of Computer Sciencn, 1982, 80-91.

[3] S.Micali, C.Rackoff, and B.Sloan. The notion of security for probabilistic cryptosystems, CRYPTO 86,31 .

[4] R.Rivest, A.Shamir, and L.Adleman, A method for obtaining digital signatures and public key cryptosystems, Comm. ACM, 21 (1978), 120-126.

[5] M.Blum and S.Goldwasser, An efficient probabilistic public-key encryption scheme which hides all partial information, CRYPTO 84, 289-299.

[6] L.Blum, M.Blum, and M.shub, A simple unpredictable pseudo-random number generator, SLAM J. Computing, 15:2, 1986, 364-383. 